\title{
On Bounds on Cumulative Teletraffic Using Min-Plus Convolution
}

\author{
Andrzej Borys
}

\begin{abstract}
Ideas and results published in two papers by R. L. Cruz in IEEE Transactions on Information Theory in 1991 gave rise to what is called now network calculus. A key role in it plays a certain inequality characterizing the behaviour of cumulative traffic curves. It defines the so-called burstiness constraint by which many kinds of trafics can be described, as for example those occurring in computer networks. Interpretation of this constraint, which can be expressed in two equivalent forms: with and without the use of min-plus convolution, can be found in papers of $\mathbf{R}$. L. Cruz. Nothing however was said about how to obtain it practically, for example, for each of representatives of a family of measured cumulative traffic curves being upperbounded. This problem is tackled in this paper, and as a result, a relation between the Cruz's constraining function and an upper-bounding function of measured traffic curves is found. The relation obtained is quite general and valid also for the case of non-fulfilment of the so-called sub-additivity property by traffic curves. For the purpose of its derivation, a notion of sub-additivity property with some tolerance $\Delta$ was introduced, and the corresponding theorem exploiting it formulated and proved. Further, to complement discussion of the above relation, a minimal burstiness constraint was added to the original Cruz's inequality and related with a lower bound of a family of measured cumulative traffic curves. The derivations presented in this paper are illustrated by examples.
\end{abstract}

Keywords-Network calculus, bounds on cumulative teletraffic curve, min-plus convolution.

\section{INTRODUCTION}

I $\mathrm{N}$ a paper [1] by R. L. Cruz, a class of traffics was defined that obey a certain burstiness constraint. It says that the difference between the cumulative traffics taken at any two time instants can not exceed the value of a certain function calculated for the difference of the aforementioned time instants. This function plays a role of an envelope for the cumulative traffic curves of a class considered [2], [3]. Moreover, the condition formulated can be expressed equivalently with the use of the so-called min-plus convolution [2], [4].

The ideas presented in two papers of Cruz [1], [5], published in 1991, together with those presented by Parekh and Gallager in [6], [7] two years later, turned out to be very fruitful. Their powerfulness followed just from a simple means of describing network traffic through the burstiness constraint [1], [5] connected with an elegant means of expressing traffic servicing with the use of the so-called service curve [6], [7]. After the pioneering works of Cruz, Parekh, and Gallager

A. Borys is with the Institute of Telecommunications, Faculty of Telecommunications and Electrical Engineering, University of Technology and Life Sciences (UTP), S. Kaliskiego 7, 85-789 Bydgoszcz, Poland (e-mail: andrzej.borys@utp.edu.pl). mentioned above, many articles appeared in the subsequent years, in which their original methodologies were further developed. This resulted in a number of new important theoretical outcomes, and in developing many new access control and scheduling algorithms for network protocols.

To get some compact information about all these achievements, an interested reader is referred to two well-written surveys [8], [9]. And after reading these tutorials, if one wishes to acquire more detailed knowledge of the solutions developed, she/he is advised to read the corresponding original papers, which are exhaustively listed in the references sections of [8] and [9].

A new branch of networking, which was meanwhile named deterministic network calculus, rose in the first phase of development of the theory and accompanying techniques having their beginning in the papers of Cruz, Parekh, and Gallager. Now, we know that the deterministic network calculus has some important drawbacks, of which the most unpleasant are too pessimistic guarantees it provides for delays, backlogs, and other parameters. The detailed explanation and discussion of these drawbacks can be found in surveys [8], [9]. Introduction of stochastic processes to the purely deterministic network calculus was a remedy for its aforementioned disadvantages and led it into the second phase of development. This originated in the so-called stochastic network calculus [9]. Nowadays, the deterministic network calculus is viewed as a matured theory, opposite to its complement, the stochastic one, of which development is still not finished. However, on the other hand, the fact of existence of a couple of textbooks on these calculi witnesses a high level of maturity of both of them. With respect to this, we mention a fundamental textbook by Le Boudec and Thiran [2], covering almost all aspects of the deterministic calculus theory. Further, note a textbook [10] by Chang devoted partly to the former theory as well as to the early approaches of the stochastic network calculus. And finally, let us mention the third one [11], written by Jiang and Liu, which deals with the problems of stochastic network calculus.

The considerations presented in this paper, on bounds on cumulative traffic using min-plus convolution, belong to the area of deterministic network calculus. They witness the fact that even in this so highly matured theory there still exist some important problems not dealt with or opened.

For example, note that nothing was said in [1] about how to relate Cruz's burstiness constraint with an upper-bound posed on a family of measured cumulative traffic curves. In this paper, we show that each of the elements of the above set of traffic curves can be characterized using the Cruz's 
constraining function related directly with an upper-bounding function of this set. The relation between the above functions is quite general and valid also for the case of non-fulfilment of the so-called sub-additivity property by traffic curves. To derive it, a new property of sub-additivity with some tolerance $\Delta$ was introduced here, and then an useful theorem exploiting it formulated and proved. Moreover, to complement discussion of the above relation, a minimal burstiness constraint was added to the original Cruz's inequality and related with a lower bound of a family of measured cumulative traffic curves.

This paper is organized as follows. After brief presentation of the problem considered and paper's objective, we discuss and present in detail in Section II mathematical formulation of the problem. In the next section, the basic result is derived. Further, Section IV presents the results illustrating how to get the Cruz's burstiness constraining function defined in [1] and a complementing minimal one, defined here, for the simplest means of bounding a set of measured cumulative traffic curves. Concluding remarks are summarized in Section V.

\section{Problem Formulation}

We say that a function $f$ is wide-sense increasing if and only if $f(s) \leq f(t)$ holds for all $s \leq t$. Consider now the functions possessing the above property and having, additionally, values identically equal to zero for negative times (that is for $t<0$ ). Such the functions belong to a class of functions named in [2] a $\mathcal{F}$-class; in [4], they were called causal processes.

Let us now take into account the curves shown in Fig. 1. We say that they belong to class $\mathcal{F}$. However, as depicted in in Fig. 1, they are, strictly saying, $\mathcal{F}$-class functions for the times $t \leq t_{\max }$, and not specified in the range $t>t_{\max }$.

In Fig. 1, $R(t)$ stands for a representative taken from a set of cumulative traffics considered. These are such functions of which values for the consecutive time instants denote numbers of bits arrived at a teletraffic node in the period from time instant 0 to time instant $t$. We will assume in what follows that $\{R(t)\}$ stands for a whole set of traffics considered, but its element (traffic representative) is denoted as $R(t)$. Further, we will assume throughout this paper that the above set of traffics is lower- and upper-bounded. That is for each of its representatives the following holds: $\beta(t) \leq R(t) \leq \alpha(t)$ for

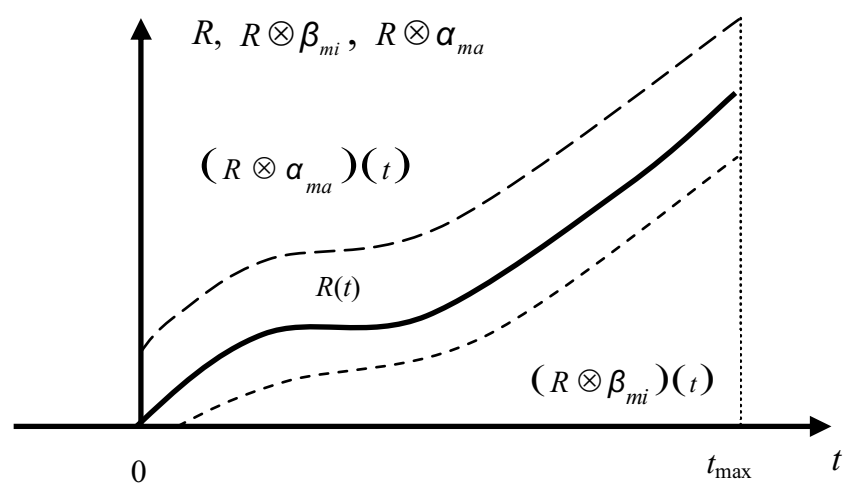

Fig. 1. A representative of cumulative traffic $R(t)$ and its minimal and maximal bounding curves. $t \leq t_{\max }$, where $\beta(t)$ and $\alpha(t)$ are some bounding functions (belonging to the $\mathcal{F}$-class defined above).

Further, observe that other functions, different from $\beta(t)$ and $\alpha(t)$, are used in Fig. 1 for lower- and upper-bounding the cumulative traffic representative $R(t)$, namely, the following ones: $\left(R \otimes \beta_{m i}\right)(t)$ and $\left(R \otimes \alpha_{m a}\right)(t)$, which are dependent upon the form of $R(t)$. They constitute its minimal and maximal bounding curves, respectively. That is the following inequality

$$
\left(R \otimes \beta_{m i}\right)(t) \leq R(t) \leq\left(R \otimes \alpha_{m a}\right)(t)
$$

holds (pointwise) for each $t$ taken from $\left\langle 0, t_{\max }\right\rangle$. The time period $\left\langle 0, t_{\max }\right\rangle$ means here the time interval in which we register (measure or/and observe) a given traffic with $t_{\max }$ being the maximal time of traffic registration. Moreover, the symbol $\otimes$ in (1) stands for the convolution operation in the min-plus algebra. It is defined as [2], [4]

$$
\left(R \otimes \beta_{m i}\right)(t)=\inf _{0 \leq s \leq t}\left(R(s)+\beta_{m i}(t-s)\right)
$$

where $s$ represents an auxiliary time variable and inf means performing the mathematical operation of finding infimum value. Similarly, we calculate the convolution of the functions $R(t)$ and $\alpha_{m a}(t)$, and of course of any other two functions.

In (1), the functions $\beta_{m i}(t)$ and $\alpha_{m a}(t)$ are assumed to be some functions (generally different from $\beta(t)$ and $\alpha(t)$ ) belonging to class $\mathcal{F}$, which enable expressing the bounding curves for the cumulative traffic function $R(t)$ - belonging also to class $\mathcal{F}$ - just in such a form that uses min-plus convolution. As this assumption is not obvious, we will discuss it again, in the next section.

It is worth noting that such the form of expressing bounding functions as in (1), with the use of min-plus convolution, is now popular in the literature on network calculus. Its original form as it occurs, for example, in the paper of Cruz [1] looks like

$$
R(t)-R(s) \leq \alpha_{m a}(t-s)
$$

and holds for every pair $t, s \in\left\langle 0, t_{\max }\right\rangle$ such that $s \leq t$. This inequality is equivalent to the right-hand side inequality in (1), that is to

$$
R(t) \leq\left(R \otimes \alpha_{m a}\right)(t) .
$$

Inequalities (3a) and (3b) express equivalently the burstiness constraint posed on the traffic $R(t)$, by its characterization through the function $\alpha_{m a}(t)$, named arrival curve in [2] or envelope function in [3], [4]. Equivalence of the descriptions (3a) and (3b) was shown in [2], [4]. Moreover, it was proved in [2] that if we calculate the convolution of two functions belonging to class $\mathcal{F}$ then the resulting one belongs to $\mathcal{F}$, too.

We include in this paper the traffic lower-bounding inequality (see the left-hand side inequality in (1)) to complement our theoretical considerations regarding (3b). In other words, in our view, the traffic characterization by (1) is simply more complete than that using exclusively (3b).

Observe also that description of the traffic representative $R(t)$ as sketched in Fig. 1 and expressed analytically by (1) can be viewed as the operation of building a bounding pipe around this traffic representative. Furthermore, note that the 
bounding pipes constructed in such a way - for different traffic representatives $R(t)$ - differ from each other.

Similarly, the traffic characterization by the following inequalities: $\beta(t) \leq R(t) \leq \alpha(t)$ valid for all time instants $t \leq t_{\max }$ and for all traffic representatives of a set of traffics considered can be interpreted as a bounding pipe. Note, however, that in this case we have to do with only a single pipe for all the traffic representatives. Obviously, this is opposite to the previous case.

Such the visualizations as described above will be helpful in further considerations presented in this paper.

The main objective of this paper is to find relations between the functions $\beta(t)$ and $\alpha(t)$ (lower- and upperbounding a set of considered cumulative traffics $\{R(t)\})$ and the functions $\beta_{m i}(t)$ and $\alpha_{m a}(t)$ used in the corresponding bounding expressions on the left- and right-hand sides of (1) (for bounding an element $R(t)$ taken from the above set $\{R(t)\})$.

It was shown in [4] that $\delta(t)$ given by

$$
\delta(t)=0 \text { for } t<0 \text { and } \infty \text { for } t \geq 0
$$

is an identity element of the convolution operation. That is it fulfills the following identity: $R \otimes \delta \equiv \delta \otimes R \equiv R$ [4] for any function $R \in \mathcal{F}$. If we use it in the obvious equality

$$
R(t)=R(t)=R(t)
$$

we get

$$
(R \otimes \delta)(t)=R(t)=(R \otimes \delta)(t)
$$

Comparison of (5b) with (1) allows us to write $\alpha_{m a}=\beta_{m i}=\delta$. This is a particular solution, which however is by no means interesting and satisfying. We look for such ones that are different from each other, and different also from $\delta$. In other words, we are interested in describing any real traffic as visualized in Fig. 1.

Before beginning derivation of the relations which hold between the functions $\beta(t)$ and $\beta_{m i}$, and similarly, between $\alpha(t)$ and $\alpha_{m a}(t)$, consider an interesting problem of determination of the characteristic of an input traffic at a traffic device (system) (in the form, for example, as given by (1)) under assumption of knowledge of its output characteristic (given, for example, by its envelope) and the traffic device (system) service curve. At this point, it is worth noting that the answer to a similar problem in classical linear systems theory is positive, opposite to the traffic devices (systems) analyzed in this paper. In the former, the answer is unique and expressed, in the frequency domain, by Fourier transform of system output signal divided by system's transmittance.

For analysis of the related problem with the use of network calculus, consider Fig. 2 depicting a teletraffic device offering a service curve $B(t)$ [2], [4], [6]. It possesses one input, with the cumulative traffic $R(t)$ applied to it, and one output at which the cumulative traffic $R^{*}(t)$ is registered. The input traffic is transferred to the device output according to a rule determined by $B(t)$. Furthermore, we assume that there exists an envelope $E(t)$ for the output traffic $R^{*}(t)$, defined as shown in (3a) or (3b) (where we put now $R^{*}$ instead of $R$ and $E$ instead of $\alpha_{m a}$, respectively). Using the definitions: of the

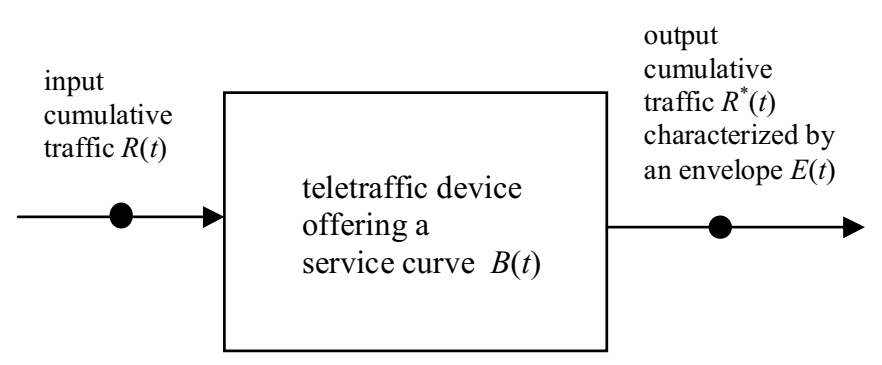

Fig. 2. Teletraffic device with one input and one output.

service curve as given, for example, in [2], [4], [6] and of the envelope [4], we can write the following

$$
(R \otimes B)(t) \leq R^{*}(t) \leq\left(R^{*} \otimes E\right)(t)
$$

for the teletraffic device of Fig. 2. (Simply in (6), the lefthand side and right-hand side inequalities constitute the corresponding definitions: of the service curve (defining the relation between input $R$ and output $R^{*}$ traffics) and of the envelope of output traffic $R^{*}$, respectively.)

As the form of (6) resembles that of (1), we can ask now whether it would be possible to get (1) from (6). To check this, assume, quite formally, $B(t)=\beta_{m i}(t)$ and $E(t)=\alpha_{m a}(t)$ in (6) and recall the causality condition

$$
R^{*}(t) \leq R(t)
$$

(which any causal traffic must obey). Taking into account (7) in (6), for eliminating $R^{*}$, we arrive at

$$
\left(R \otimes \beta_{m i}\right)(t) \leq\left(R \otimes \alpha_{m a}\right)(t)
$$

and

$$
\left(R \otimes \beta_{m i}\right)(t) \leq R(t) .
$$

Note that in getting (8a) a rule was used that if (7) holds then $R^{*} \otimes \alpha_{m a} \leq R \otimes \alpha_{m a}$ does, too. This rule was proved, for example, in [2] (rule 10 on page 115 therein).

Comparison of inequalities (8a) and (8b) with those in (1) shows that they are not the same (identical). So we conclude that derivation of (1) from (6) is not possible.

Finally, we remark that as $B=E$ holds for teletraffic devices called greedy shapers [2], [12] or regulators with causal subadditive envelope [4], this simplifies (8a) and (8b) to $\left(R \otimes \beta_{m i}\right)(t)=\left(R \otimes \alpha_{m a}\right)(t) \leq R(t)-$ under the previous assumption of $\beta_{m i}(t)=B(t)$ and $\alpha_{m a}(t)=E(t)$. However, this result does not mean the equivalence with (1) because there are still such times for which $\left(R \otimes \alpha_{m a}\right)(t)<R(t)$, what contradicts the right-hand side of (1).

\section{DERIVATION OF (1)}

In the derivation of (1) presented here, we restrict ourselves to a subclass of functions $\beta(t), \beta_{m i}(t), \alpha(t), \alpha_{m a}(t)$, and $R(t)$, belonging to $\mathcal{F}$, that are right-continuous. Moreover, our traffic observation (registration) time will be restricted to an interval of finite length $\left\langle 0, t_{\max }\right\rangle$. Furthermore, we will not consider times $t<0$, for which obviously $\beta(t)=\alpha(t)=R(t)=0$ holds, and we will not be interested 
a)

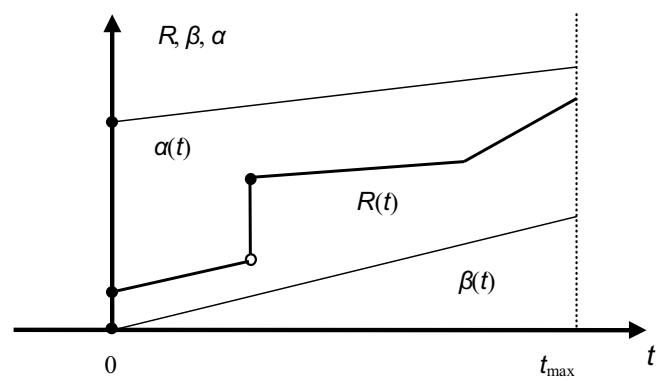

b)

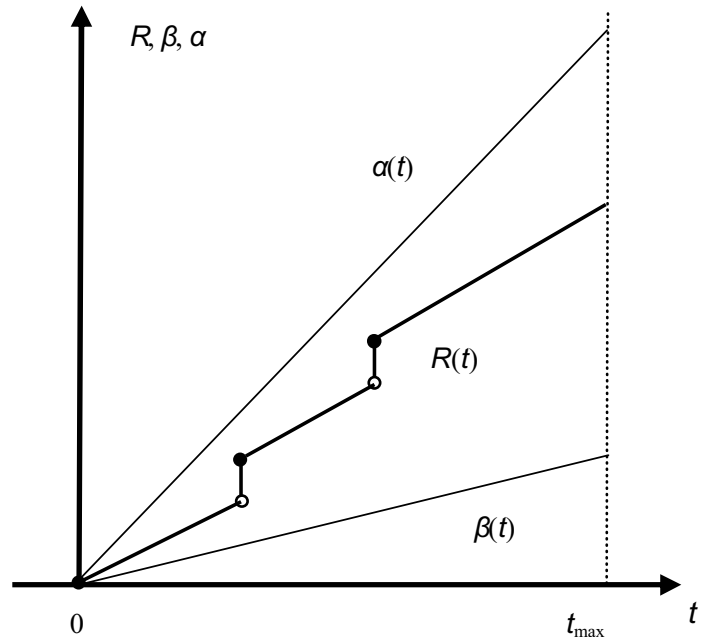

Fig. 3. Cumulative traffic description with lower $\beta(t)$ and upper $\alpha(t)$ bounding functions for two cases: a) when $R(0) \neq 0$ and b) when $R(0)=0$.

in times $t>t_{\max }$, for which, we assume, the above functions are not specified. Additionally, we will assume that the functions $\beta(t)$ and $\alpha(t)$ are continuous in the open interval $\left(0, t_{\max }\right)$ (that is they are left- as well as right-continuous in each point of this interval). However, we will allow that they are only right-continuous at the point $t=0$. In other words, at this point, the functions $\beta(t)$ and $\alpha(t)$ will be eventually discontinuous. Furthermore, it will be assumed that the function $R(t)$ may be discontinuous at the point $t=0$ and at the points of the interval $\left(0, t_{\max }\right)$. However, the number of such discontinuities of $R(t)$ will be always finite.

This description as sketched above is illustrated in Fig. 3 by two typical situations which can occur: (a) when $R(0) \neq 0$ and (b) when $R(0)=0$.

Complementing the above description and Fig. 3, observe that the functions $\beta(t), \alpha(t)$, and $R(t)$ possess only the left-continuity property at the point $t_{\max }$ (or, in the case of $R(t)$, the continuity property may be even not specified). This follows from two facts: that the functions $\beta(t), \alpha(t)$, and $R(t)$ are assumed to be not specified for times $t>t_{\max }$, and that their continuity and discontinuity properties for times $t<t_{\max }$ are as stated above.

And finally at this point, we remark that the functions derived from $\beta(t), \alpha(t)$, as $\beta_{m i}(t), \alpha_{m a}(t)$, and $\beta_{z}(t), \alpha_{\Delta}(t)$, (the latter two will be introduced in this section) inherit all the properties of $\beta(t), \alpha(t)$ discussed above.

Now, observe that the way of bounding a family of cumu- lative traffic curves as shown in Fig. 3 is the simplest one because it uses segments of straight lines. This idea, of using piece-wise linear curves, originates from the choices made for envelope functions of simple regulators, see for example [4]. Of course, the usage of more sophisticated bounding functions is possible in our approach.

The lower $\beta(t)$ and upper $\alpha(t)$ bounding functions shown in Fig. 3a have, analytically, the following form:

$$
\beta(t) \Rightarrow \beta_{1}(t)= \begin{cases}0 & \text { for } t<0 \\ \rho_{\beta 1} t+\sigma_{\beta 1}=\rho_{\beta 1} t & \text { for } 0 \leq t \leq t_{\max } \\ \text { not specified } & \text { for } t>t_{\max }\end{cases}
$$

and

$$
\alpha(t) \Rightarrow \alpha_{1}(t)= \begin{cases}0 & \text { for } t<0 \\ \rho_{\alpha 1} t+\sigma_{\alpha 1} & \text { for } 0 \leq t \leq t_{\max } \\ \text { not specified } & \text { for } t>t_{\max }\end{cases}
$$

where $\sigma_{\beta 1}=0, \rho_{\alpha 1}>\rho_{\beta 1}>0$, and $\sigma_{\alpha 1}>0$ are constant coefficients. Note that a special choice for $\beta(t)$ in Fig. 3a was made with the coefficient $\sigma_{\beta 1}$ equal to zero. Evidently, other choices are also possible.

Similarly, for the case of Fig. 3b, we have

$$
\beta(t) \Rightarrow \beta_{2}(t)= \begin{cases}0 & \text { for } t<0 \\ \rho_{\beta 2} t & \text { for } 0 \leq t \leq t_{\max } \\ \text { not specified } & \text { for } t>t_{\max }\end{cases}
$$

and

$$
\alpha(t) \Rightarrow \alpha_{2}(t)= \begin{cases}0 & \text { for } t<0 \\ \rho_{\alpha 2} t & \text { for } 0 \leq t \leq t_{\max } \\ \text { not specified } & \text { for } t>t_{\max }\end{cases}
$$

where, as before, $\rho_{\alpha 2}>\rho_{\beta 2}>0$, mean some constant coefficients. Moreover, in (9-12), the following notational convention was applied: the functions $\beta(t)$ and $\alpha(t)$ referring to the cases depicted in Figs. $3 \mathrm{a}$ and $3 \mathrm{~b}$ have the indices 1 and 2 , respectively

Fig. 3 shows that, independently of the case: a) or b), we can express the relation between $\beta(t), \alpha(t)$ and $R(t)$ as

$$
\beta(t) \leq R(t) \leq \alpha(t) \text { for } t \leq t_{\max }
$$

and assume it as "not specified" for times $t>t_{\max }$. Moreover, without loss of generality, we can consider further (13) only for times restricted to the interval $\left\langle 0, t_{\max }\right\rangle$.

Let us now introduce in (13) auxiliary variables $t^{\prime}$ and $s$, assuming values from the interval $\left\langle 0, t_{\max }\right\rangle$, and related with the variable $t$ as follows

$$
t=t^{\prime}-s \geq 0
$$

Applying (14) in (13) and renaming then, for simplicity of notation, the auxiliary variable $t^{\prime}$ as $t$ gives

$$
\beta(t-s) \leq R(t-s) \leq \alpha(t-s)
$$

for $t-s \geq 0$ and $t, s \in\left\langle 0, t_{\max }\right\rangle$. In the next step, adding $R(s)$ on both the sides of $(15)$ and to the expression in the middle in (15), we obtain

$$
R(s)+\beta(t-s) \leq R(s)+R(t-s) \leq R(s)+\alpha(t-s) .
$$


Assume now $\beta(t)$ and $\alpha(t)$ in (16) so situated as in Fig. 3a, with the additional property that $R(0)=0$. Next, consider the left-hand side inequality in (16). For this case, we can write

$$
R(0)+\beta_{z}(t) \leq R(0)+R(t)=R(t)
$$

where $\beta_{z}$ is used for denoting $\beta$, occurring in (13), in a special case when $R(0)=0$ holds.

Furthermore, because of the assumed properties of the functions $\beta(t)$ and $R(t)$, the following

$$
\lim _{s \rightarrow 0^{+}}(R(s)+\beta(t-s))=R(0)+\beta(t)
$$

holds, where $\lim _{s \rightarrow 0^{+}}$means the right-hand side limit at the point $s=0$. Using this, (17) and the definition of the infimum operation, we can write

$$
\begin{gathered}
\inf _{0 \leq s \leq t}\left(R(s)+\beta_{z}(t-s)\right) \leq \lim _{s \rightarrow 0^{+}}\left(R(s)+\beta_{z}(t-s)\right)= \\
=R(0)+\beta_{z}(t) \leq R(t) .
\end{gathered}
$$

That is, we obtain finally

$$
\inf _{0 \leq s \leq t}\left(R(s)+\beta_{z}(t-s)\right) \leq R(t)
$$

or in a more compact form

$$
\left(R \otimes \beta_{z}\right)(t) \leq R(t)
$$

valid for all the times $t$ from the interval $\left\langle 0, t_{\max }\right\rangle$.

Observe that $(19 \mathrm{c})$ represents the left-hand side inequality in (1) with $\beta_{m i}(t)=\beta_{z}(t)$.

For the case of $R(0) \neq 0$, we get a similar result. To show this, we will use the previous result (19b) for an auxiliary traffic $P(t)=R(t)-R(0)$ that fulfills the requirement of $P(0)=0$ and is lower-bounded by some $\beta_{z}(t)$ (that is $\beta_{z}(t) \leq P(t)$ holds). So substituting $P(t)$ instead of $R(t)$ in (19b) gives

$$
\inf _{0 \leq s \leq t}\left(P(s)+\beta_{z}(t-s)\right) \leq P(t) .
$$

From (20a), using $P(t)=R(t)-R(0)$, we get

$$
\inf _{0 \leq s \leq t}\left(R(s)-R(0)+\beta_{z}(t-s)\right) \leq R(t)-R(0) .
$$

and moving the constant value $R(0)$ outside the operation infimum, we arrive finally at

$$
\inf _{0 \leq s \leq t}\left(R(s)+\beta_{z}(t-s)\right) \leq R(t) .
$$

Note now that (20c) is identical with (19b) and its equivalent $(19 c)$. So, similarly as before, we conclude that (20c) represents the left-hand side inequality in (1) with $\beta_{m i}(t)=\beta_{z}(t)$. However, in opposite to the previous case, the function $\beta_{z}(t)$ is not now, in general, the same function $\beta(t)$ that was chosen originally for bounding $R(t)$ by writing (13). The following sequence of inequalities explains the above fact

$$
\begin{gathered}
\beta(t) \leq R(t) \Rightarrow \\
\beta_{z}(t)=\beta(t)-R(0) \leq R(t)-R(0)=P(t) .
\end{gathered}
$$

Note that the function $\beta_{z}(t)$ defined by (21) differs from $\beta(t)$ by a constant $R(0)$. Observe also that this function can eventually have negative values in some time interval beginning at $t=0$. On the other hand, we want such the functions to be nonnegative as the traffic cumulative functions are [2]. In the case considered, it is easy to fulfill this requirement by choosing - instead of $\beta_{z}(t)$ defined in (21) - the following function

$$
\beta_{z}(t)=[\beta-R(0)]^{+}
$$

where the symbol $[x(t)]^{+}$denotes the operation of finding maximum value in the set $\{x(t), 0\}$ for each time instant [9] It can be easily shown that the function $\beta_{z}(t)$ given by (22) fulfills the requirement: $\beta_{z}(t) \leq P(t)$. Moreover, we have $\beta_{z}(t) \leq \beta(t) \leq R(t)$. And this means, generally, that we have to use in (20c) the traffic lower-bounding function that is not so tight as that originally chosen in (13). However, we draw the reader's attention to the fact that it is possible to choose $\beta(t)$ in (13) in a way (not so tight) guaranteeing that not only (13) is fulfilled, but also the following inequality: $\beta(t) \leq R(t)-R(0)=P(t)$. Then, such the $\beta$ can be also treated as $\beta_{z}$, and used in (20c).

Consider now the right-hand side of (16). Obviously, this inequality results in $R(t) \leq \alpha(t)$ for $s=0$ and $R(0)=0$, however, it is not possible to draw the conclusion from it that $R(t) \leq \alpha(t) \leq(R \otimes \alpha)(t)$. We must find another way of showing that the right-hand side inequality in (1) can be derived from the inequality $R(t) \leq \alpha(t)$. As it will be shown below, we achieve this by posing a certain condition on the traffic cumulative function $R(t)$; it is the property of subadditivity [2], [4]. So, we require that $R(t)$ fulfills the following inequality

$$
R(t) \leq R(s)+R(t-s)
$$

for all the pairs $t, s \in\left\langle 0, t_{\max }\right\rangle$ such that $s \leq t$.

Using (23) in (16) gives

$$
R(t) \leq R(s)+R(t-s) \leq R(s)+\alpha(t-s)
$$

valid for all the times $t \in\left\langle 0, t_{\max }\right\rangle$ and with $s$ changing for a fixed $t$ in the range $\langle 0, t\rangle$. Therefore, we can write

$$
R(t) \leq \inf _{0 \leq s \leq t}(R(s)+\alpha(t-s)) .
$$

Moreover, similarly as the result (19c), (24b) can be also written in a compact form as

$$
R(t) \leq(R \otimes \alpha)(t) .
$$

Observe that $(24 \mathrm{c})$ represents the right-hand side inequality in (1) with $\alpha_{m a}(t)=\alpha(t)$.

It can happen, however, that a given traffic is not subadditive, i.e. it does not satisfy (23). Nevertheless, as we show here, it possesses always a property named "subadditivity with tolerance $\Delta$ ". This property is formulated and proved in Theorem 1 below.

Theorem 1: Let be a teletraffic characterized by a cumulative traffic function that is a $\mathcal{F}$-class function for the times $t \leq t_{\max }$, and eventually not specified in the range $t>t_{\max }$. Further, let be this function bounded. That is $R(t) \leq M$, with $M$ being a constant, holds for the times $t \leq t_{\max }$. Then, the following

$$
R(t) \leq R(s)+R(t-s)+\Delta_{\infty}
$$


is valid for all the pairs $t, s \in\left(-\infty, t_{\max }\right\rangle$ such that $s \leq t$. Moreover, similarly in the case of a finite interval $\left\langle 0, t_{\max }\right\rangle$, we have

$$
R(t) \leq R(s)+R(t-s)+\Delta
$$

i.e. for all the pairs $t, s \in\left\langle 0, t_{\max }\right\rangle$ such that $s \leq t$.

In (25a) and (25b), $\Delta_{\infty}$ and $\Delta$, respectively, are certain constants that can be interpreted as tolerances posed on fulfilment of the subadditivity requirement by $R(t)$ in the corresponding intervals defined above.

Proof: Let hold the following

$$
R(t) \geq R(s)+R(t-s)
$$

for some pairs $t, s \in\left(-\infty, t_{\max }\right\rangle$ such that $s \leq t$. This means that the value of the difference $R(t)-R(s)-R(t-s)$ for these pairs of $t$ and $s$ is greater than zero or equals zero. Let us now take into account the bound for the greatest value of the above difference, that is

$$
\sup _{\left.-\infty, t_{\max }\right\rangle, s \leq t}(R(t)-R(s)-R(t-s))=\Delta_{\infty}
$$

This bound is denoted in (27) as $\Delta_{\infty}$. Moreover, it follows from the boundness of the function $R(t)$ for $t \in\left(-\infty, t_{\max }\right\rangle$ that $\Delta_{\infty}$ always exists, is finite and nonnegative. Further, we can write now

$$
\begin{gathered}
R(t)-R(s)-R(t-s) \leq \Delta_{\infty} \Rightarrow \\
R(t) \leq R(s)+R(t-s)+\Delta_{\infty} .
\end{gathered}
$$

Observe that the proof for a finite interval $\left\langle 0, t_{\max }\right\rangle$ goes along the same lines as above, however, now with

$$
\Delta=\sup _{t, s \in\left\langle 0, t_{\max }\right\rangle, s \leq t}(R(t)-R(s)-R(t-s))
$$

instead of $\Delta_{\infty}$. Furthermore, the equivalent of (28) for the finite interval $\left\langle 0, t_{\max }\right\rangle$ has the form

$$
R(t) \leq R(s)+R(t-s)+\Delta
$$

and this ends the proof.

Using (25b) in (16) gives

$$
R(t)-\Delta \leq R(s)+R(t-s) \leq R(s)+\alpha(t-s)
$$

and finally

$$
R(t) \leq R(s)+\alpha(t-s)+\Delta
$$

valid for all the times $t \in\left\langle 0, t_{\max }\right\rangle$ and with $s$ changing for a fixed $t$ in the range $\langle 0, t\rangle$. Hence, $(31 \mathrm{~b})$ can be also put into a more compact form using the min-plus convolution operation

$$
R(t) \leq(R \otimes \alpha)(t)+\Delta=\left(R \otimes \alpha_{\Delta}\right)(t)
$$

where the modified bounding function $\alpha_{\Delta}(t)$ is given by

$$
\alpha_{\Delta}(t)=\alpha(t)+\Delta .
$$

Observe that $(31 \mathrm{c})$ represents the right-hand side inequality in (1) with $\alpha_{m a}(t)=\alpha_{\Delta}(t)$.

Concluding this section, note that inequalities (20c) and (31c) provide for the general case the corresponding bounding functions related with those in (1). In this case, the functions $\beta_{m i}(t)$ and $\alpha_{m a}(t)$ occurring in (1) are not identical with the bounding functions $\beta(t)$ and $\alpha(t)$ chosen in (13). The latter ones must be then slightly modified to be used in (1). As shown, the modifications are related with the existence of the nonzero value of $R(0)$ and the lack of fulfilment of the subadditivity condition by $R(t)$. Moreover, it was also shown that, when $R(0)=0$ and the function $R(t)$ is subadditive, there is no need for the aforementioned modifications.

Finally, it is worth noting that we needed to ask whether $R(0)$ was equal to zero or not in our considerations regarding the left-hand side inequality in (1). In this case, however, we did not need to ask whether $R(t)$ fulfilled the subadditivity condition or not. And the reverse took place in the considerations regarding the right-hand side inequality in (1).

\section{DisCUSSION AND EXAMPLES}

For illustration of the results derived in the previous section, let us consider the curve of the cumulative traffic $R(t)$ sketched in Fig. 3a. We find the functions $\beta_{m i}(t)$ and $\alpha_{m a}(t)$ for it for bounding in the sense of (1). As we already know from Section III, the functions $\beta(t)$ and $\alpha(t)$ depicted in Fig. 3a and expressed analytically by (9) and (10) can not be applied directly. They must be modified because of two reasons: the discontinuity of $R(t)$ at $t=0$ and its presumable non-subadditivity. We begin with $\beta(t)$, and observe that according to (22), we have to apply now

$$
\begin{aligned}
& \beta_{z 1}(t)=\left[\beta_{1}(t)-R(0)\right]^{+}=\left[\rho_{\beta 1} t-R(0)\right]^{+}= \\
= & \begin{cases}0 & \text { for } 0 \leq t \leq R(0) / \rho_{\beta 1} \\
\rho_{\beta 1} t-R(0) & \text { for } R(0) / \rho_{\beta 1} \leq t \leq t_{\max }\end{cases}
\end{aligned}
$$

for the range $\left\langle 0, t_{\max }\right\rangle$. Finally, this gives $\beta_{m i}(t)=\beta_{z 1}(t)$.

It is really difficult to evaluate $R(t)$, obtained for instance in traffic measurements, whether it is subadditive or not. One way of doing this is a skilful curve inspection that provides better or worse estimates of the parameter $\Delta$. We explain this point on an example of $R(t)$ sketched in Fig. 3a. Observing $R(t)$ in Fig. 3a and having in mind (29), we guess that the greatest positive value of the difference $R(t)-R(s)-R(t-s)$ occurs when $R(t)$ is taken from the second segment of the piecewise linear curve describing the traffic in the range $\left\langle 0, t_{\max }\right\rangle$, but at the same time, $R(s)$ and $R(t-s)$ lie in its first segment. That is they lie before the occurrence of the discontinuity, taking place, say, at time $t_{d}$. Let us denote the magnitude of discontinuity at this time instant as $\sigma_{d}$; it equals

$$
\sigma_{d}=R\left(t_{d}\right)-\lim _{t \rightarrow t_{d}^{-}} R(t)
$$

where $\lim _{t \rightarrow t_{d}^{-}}$means the left-hand side limit at the point $t=$ $t_{d}$. Moreover, denote the line slopes in the first and second segments as $a_{1}$ and $a_{2}$, respectively. We observe in Fig. 3a that $a_{1}>a_{2}$.

Applying these notations, we can describe the lines of the aforementioned curve pieces in the following way

$$
\begin{gathered}
R(s)=a_{1} s+R(0) \text { for } 0<s<t_{d} \\
R(t-s)=a_{1}(t-s)+R(0) \text { for } 0<t-s<t_{d} \\
R(t)=a_{2}\left(t-t_{d}\right)+a_{1} t_{d}+R(0)+\sigma_{d} \text { for } t_{d} \leq t<2 t_{d} .
\end{gathered}
$$


Using then (34a-c) to express $R(t)-R(s)-R(t-s)$, after some algebraic manipulations, we get

$$
R(t)-R(s)-R(t-s)=-\left(a_{1}-a_{2}\right)\left(t-t_{d}\right)+\sigma_{d}-R(0)
$$

valid for all $0<s<t_{d}$ and $t_{d} \leq t<2 t_{d}$. As $a_{1}-a_{2}>0$ and $t-t_{d} \geq 0$ hold, (35) assumes the largest value for $t=t_{d}$, and equals $\sigma_{d}-R(0)$. Fig. 3a shows that this value is positive, witnessing for the non-subadditivity of the traffic curve $R(t)$. Furthermore, it is the value of the parameter $\Delta$. Note however that, generally, it is difficult to obtain an exact value of $\Delta$ using our "inspection approach". Nevertheless, we get a better or worse estimate of $\Delta$ in each case.

Using the above result and expressions (10) and (31d), we obtain for $\alpha_{\Delta}(t)$

$$
\alpha_{\Delta 1}(t)=\rho_{\alpha 1} t+\sigma_{\alpha 1}+\sigma_{d}-R(0)
$$

in the example considered for the range $\left\langle 0, t_{\max }\right\rangle$, for upperbounding the traffic according to (31c). Finally, this gives

$$
\alpha_{m a 1}(t)=\alpha_{\Delta 1}(t) .
$$

Note that there are many possible choices for associating bounding functions with the cumulative traffic curves. We will now present another choice, a little bit different from that discussed just before. To this end, assume that we have to do with a cumulative traffic function $R(t)$ for which $R(0) \neq 0$ holds. We associate with this traffic an auxiliary one, named $P(t)$, by calculation of $P(t)=R(t)-R(0)$ for each $t$. Then, we sketch the bounding functions as depicted in Fig. 3b with $R(t)$ replaced now by $P(t)$. Denoting them as $\beta_{z 2}(t)$ and $\alpha_{2}(t)$, we have the following descriptions

$$
\beta_{z 2}(t)=\rho_{\beta_{2}}(t)
$$

and

$$
\alpha_{2}(t)=\rho_{\alpha_{2}}(t)
$$

in the range $\left\langle 0, t_{\max }\right\rangle$; their descriptions in two other intervals: $(-\infty, 0)$ and $\left(t_{\max }, \infty\right)$ are the same as the previous ones (compare (9) with (11) and (10) with (12)).

Observe now that because $P(t)$ fulfills the requirement $P(0)=0$, we can use the previous result (20a). That is the following

$$
\inf _{0 \leq s \leq t}\left(P(s)+\beta_{z 2}(t-s)\right) \leq P(t)
$$

holds in the case considered. Adding afterwards $R(0)$ on both sides of inequality (39), we get

$$
\inf _{0 \leq s \leq t}\left(P(s)+\beta_{z 2}(t-s)\right)+R(0) \leq P(t)+R(0) .
$$

Further, using the fact that addition of a constant after performing the infimum operation gives the same result as in the case of adding it to the expression inside this operation, and observing that $P(t)+R(0)=R(t)$, we obtain from (40a)

$$
\inf _{0 \leq s \leq t}\left(R(s)+\beta_{z 2}(t-s)\right) \leq R(t) .
$$

We remark that (40b) is identical with (20c), however, in this case, it uses another form of the bounding function $\beta_{z}(t)$.

Consider now the upper-bounding of the traffic $P(t)$. And we begin with remark that if an original traffic $R(t)$ is subadditive then auxiliary one $P(t)=R(t)-R(0)$ is not. To show this, take into account $R(t)$ being subadditive, what means that (23) holds. Subtracting $R(0)$ on both sides of (23) allows us to rewrite it as

$$
R(t)-R(0) \leq R(s)-R(0)+R(t-s)-R(0)+R(0) .
$$

Introducing afterwards $P(t)=R(t)-R(0)$ in (41a) gives

$$
P(t) \leq P(s)+P(t-s)+R(0) .
$$

Comparison of (41b) with (25b) shows that the traffic $P(t)$ is subadditive with the tolerance $\Delta_{P}=R(0)$. So in this case, for upper-bounding the traffic $P(t)$, we must use (31c) (with $R(t)$ therein replaced now with $P(t)$ ) and with the function $\alpha_{\Delta}(t)$ having the form

$$
\alpha_{\Delta 2}(t)=\alpha_{2}(t)+R(0)=\rho_{\alpha 2} t+R(0) .
$$

Note that in derivation of (42) expressions (31d) and (38) were used.

To get the upper bound for the original traffic $R(t)$, we substitute $P(t)=R(t)-R(0)$ and $\alpha_{\Delta 2}(t)$ given by (42) into (31c), as described above. This gives

$$
R(t)-R(0) \leq\left((R(\cdot)-R(0)) \otimes\left(\alpha_{2}(\cdot)+R(0)\right)\right)(t) .
$$

Using the definition of the min-plus convolution (2), it is easy to show that the following

$$
\left((R(\cdot)-R(0)) \otimes\left(\alpha_{2}(\cdot)+R(0)\right)\right)(t)=\left(R \otimes \alpha_{2}\right)(t)
$$

holds. Applying this in (43a) and rearranging the expressions, we arrive finally at

$$
R(t) \leq\left(R \otimes \alpha_{2}\right)(t)+R(0)=\left(R \otimes \alpha_{\Delta 2}\right)(t) .
$$

Comparing (43c) with (24c), we observe that the function $\alpha_{\Delta 2}(t)=\rho_{\alpha 2}(t)+R(0)$ plays now a role of the function $\alpha_{1}(t)=\rho_{\alpha 1} t+\sigma_{\alpha 1}$ used in the first scheme of bounding the traffic $R(t)$ - as depicted in Fig. 3a. Hence, we can assume for the parameters $\rho_{\alpha 1}$ and $\sigma_{\alpha 1}$ the following values: $\rho_{\alpha 2}$ and $R(0)$, respectively. Further, note that the number of possible choices of $\rho_{\alpha 1}$ and $\sigma_{\alpha 1}$ is infinite. However, in each of them, the following condition: $\rho_{\alpha 1} \geq R(0)$ will be fulfilled, as this example and Fig. 3a show.

Consider now the case of the traffic $R(t)$ that is subadditive with tolerance, say, $\Delta_{R}>0$. The value of this tolerance is given by (29). Adding and subtracting $2 R(0)$ on the righthand side of (29), and reordering afterwards the expressions, we get

$$
\begin{gathered}
\Delta_{R}=\sup _{t, s \in\left\langle 0, t_{\max }\right\rangle, s \leq t}(R(t)-R(0)-(R(s)-R(0))- \\
-(R(t-s)-R(0)))-R(0) .
\end{gathered}
$$

Introducing then the auxiliary traffic $P(t)=R(t)-R(0)$ in (44), we obtain

$$
\sup _{t, s \in\left\langle 0, t_{\max }\right\rangle, s \leq t}(P(t)-P(s)-P(t-s))=\Delta_{R}+R(0)=\Delta_{P}
$$

for this traffic, with $\Delta_{P}$ meaning its tolerance in fulfilling the subadditivity condition. 
Proceeding analogously as before in the case of $P(t)$ having the tolerance $\Delta_{P}=R(0)$, see the results shown in expressions from (42) to (43c), we get now the equivalents of (42) and $(43 c)$ as

$$
\alpha_{\Delta_{R} 2}(t)=\alpha_{2}(t)+\Delta_{R}+R(0)=\rho_{\alpha 2} t+\Delta_{R}+R(0)
$$

and

$$
R(t) \leq\left(R \otimes \alpha_{2}\right)(t)+\Delta_{R}+R(0)=\left(R \otimes \alpha_{\Delta_{R} 2}\right)(t)
$$

respectively. Note further that conclusions we can draw from (46) and (47) are similar to those presented above for the previous case.

\section{CONCLUDING REMARKS}

The problem of lower and upper bounding of the function of cumulative traffic with the use of min-plus convolution operation has been considered in this paper. It has been shown how to get such the bounds from the bounding functions posed on a family of cumulative traffic curves obtained, for example, by performing traffic measurements. We have explained how the function used in construction of the minplus convolution lower bound is influenced by the traffic's nonzero value at time $t=0$ (the initial value). Moreover, we have shown the influence of the fact of not possessing the property of subadditivity on the form of the function used in construction of the traffic's min-plus convolution upper bound. For each of the cases mentioned, an expression determining the needed modification has been derived. Moreover, a very useful property of subbaditivity with the tolerance $\Delta$ has been defined and used in derivations.

\section{REFERENCES}

[1] R. L. Cruz, "A calculus for network delay, Part I: Network elements in isolation," IEEE Transactions on Information Theory, vol. 37, pp. 114-131, January 1991.

[2] J.-Y. Le Boudec and P. Thiran, Network Calculus. A Theory of Deterministic Queuing Systems for the Internet. Berlin: Springer Verlag, 2004.

[3] S. Mao and S. S. Panwar, "A survey of envelope processes and their applications in quality of service provisioning," IEEE Communications Surveys, vol. 8, no. 3, pp. 2-19, 2006.

[4] R. Agrawal, R. L. Cruz, C. Okino, and R. Rajan, "Performance bounds for flow control protocols," IEEE/ACM Transactions on Networking, vol. 7, pp. 310-323, June 1999.

[5] R. L. Cruz, "A calculus for network delay, Part II: Network analysis," IEEE Transactions on Information Theory, vol. 37, pp. 132-141, January 1991.

[6] A. K. Parekh and R. G. Gallager, "A generalized processor sharing approach to flow control in integrated services networks: The single node case," IEEE/ACM Transactions on Networking, vol. 1, pp. 344357, June 1993.

[7] — , "A generalized processor sharing approach to flow control in integrated services networks: The multiple node case," IEEE/ACM Transactions on Networking, vol. 2, pp. 137-150, April 1994.

[8] V. Firoiu, J.-Y. Le Boudec, D. Towsley, and Z.-L. Zhang, "Theories and models for internet quality of service," Proceedings of the IEEE, vol. 90 no. 9 , pp. $1565-1591,2002$.

[9] M. Fidler, "A survey of deterministic and stochastic service curve models in the network calculus," IEEE Communication Surveys \& Tutorials, vol. 12, no. 1, pp. 59-86, 2010.

[10] C.-S. Chang, Performance Guarantees in Communication Networks. Berlin: Springer Verlag, 2000

[11] Y. Jiang and Y. Liu, Stochastic Network Calculus. Berlin: Springer Verlag, 2008.

[12] J.-Y. Le Boudec, "Some properties of variable length packet shapers," IEEE/ACM Transactions on Networking, vol. 10, pp. 329-337, June 2002. 